\title{
La impulsividad y sistemas BIS/BAS como factores de riesgo para el consumo de drogas en personas privadas de la libertad
}

\author{
Impulsiveness and BIS / BAS systems as risk factors for drug use in people \\ deprived of Liberty
}

Marco Antonio Santana Campas

Laura Rocío Juárez García

Universidad de Guadalajara

\section{Resumen}

El consumo de drogas y la impulsividad se encuentran asociados al comportamiento criminal. Objetivo: determinar el efecto de los sistemas BIS/BAS e impulsividad en el consumo de drogas en personas privadas de la libertad. Muestra: 175 personas privadas de la libertad en una cárcel de mediana seguridad del estado de Jalisco, México. Método: investigación cuantitativa, no experimental, transversal. Instrumentos: escalas BIS/BAS, de impulsividad y cuestionario exprofeso para variables sociodemográficas. Resultados: de la muestra, la media de edad fue de $36, D E=9$, estado civil, 35.4 solteros y $27.7 \%$ concubinato. Escolaridad: $50.3 \%$ educación primaria y $24.6 \%$ educación secundaria. El $68 \%$ reportó consumo de drogas antes de ingresar a reclusión. Las variables de estudio explican el $23.5 \%$ de la varianza del consumo de drogas en personas privadas de la libertad. Las personas que vivieron violencia en la infancia $(\mathrm{OR}=4.9)$ y con autoconcepto impulsivo $(\mathrm{OR}=5.09)$ tienen mayor riesgo de consumo de drogas. Se descarta el efecto del sistema BIS sobre el consumo de drogas en personas privadas de la libertad.

Palabras clave: impulsividad, sistemas BIS/BAS, consumo de drogas, cárcel, prisionero

Nota del autor

Marco Antonio Santana Campas, Centro Universitario del Sur de la Universidad de Guadalajara. Laura Rocío Juárez García, Centro Universitario del Sur de la Universidad de Guadalajara.

La correspondencia en relación con este artículo debe dirigirse a Marco Antonio Santana Campas, Centro Universitario del Sur. Av. Enrique Arreola Silva No. 883, colonia Centro C.P. 49000, Ciudad Guzmán, Jalisco, México.

Dirección electrónica: mascampas@gmail.com

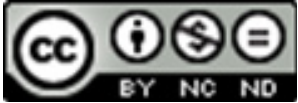




\begin{abstract}
Drug use and impulsivity are associated with criminal behavior. Objective: to determine the effect of BIS / BAS systems and impulsiveness on drug consumption in people deprived of liberty. Sample: 175 people deprived of liberty in a medium-security prison in the state of Jalisco, Mexico. Method: Quantitative, non-experimental, cross-sectional research. Instruments: BIS / BAS scales, impulsivity and express questionnaire for sociodemographic variables. Results: of the sample, the mean age was 36, $\mathrm{SD}=9$, marital status: 35.4 singles and $27.7 \%$ cohabitation. Schooling: 50.3 primary education and $24.6 \%$ middle school. $68 \%$ reported drug use before entering prison. Study variables explain $23.5 \%$ of the variance of drug use in people deprived of liberty. People who experienced violence in childhood $(\mathrm{OR}=4.9)$ and with impulsive self-concept $(\mathrm{OR}=5.09)$ have a higher risk of drug use. The effect of the BIS system on drug consumption in people deprived of liberty is ruled out.
\end{abstract}

Keywords: Impulsivity, BIS/BAS systems, drug use, jail, prisoner

La Organización Mundial de la Salud (OMS) mencionó que en el 2017 alrededor de 271 millones de personas entre los 15 y los 54 años consumieron algún tipo de droga, esto corresponde al $5.5 \%$ de la población (OMS, 2019). El consumo de drogas está asociado a conductas criminales, en la etapa inicial delictiva el $\mathrm{Ca}$ nnabis es la más consumida y en personas con trayectoria consolidada la droga de más consumo es la cocaína. Principalmente las conductas delictivas asociadas al consumo de drogas se detonan por dos situaciones: ante la falta de recursos económicos conseguidos de manera lícita para el consumo o por los problemas psíquicos derivados del consumo (Uceda-Maza et al., 2016).

En México, según la Encuesta Nacional de Consumo de Drogas, Alcohol y Tabaco (ENCODAT) de los adolescentes que cometieron algún delito bajo los efectos de alguna droga, se identificó que el alcohol (47.7 \%) fue la sustancia más utilizada bajo esta situación, seguida por los inhalables (24.6\%) y la mariguana (16.9\%) (ENCODAT, 2017). En jóvenes privados de la libertad Santana-Campas, et al. (2019) reportaron que el $24.4 \%$ habían consumido algún tipo de droga durante la realización de la conducta delictiva. En cuanto a la incidencia delictiva, el Secretariado Ejecutivo del Sistema Nacional de Seguridad Pública reportó que durante el 2019 se cometieron 1860962 delitos del fuero común y 751551 del fuero federal.

$\mathrm{Se}$ ha identificado una relación directa entre el consumo de drogas, conductas delictivas y delincuencia violenta. Además, los problemas se agravan cuando dentro de los centros penitenciarios se continúa el consumo, creando problemas de violencia, corrupción, y psiquiátricos y las conductas delictivas se perpetúan dentro de las cárceles (Castellanos, 2015; Larrotta et al., 2017; Esbec y Echeburúa, 2016). Asociado al consumo de drogas $y$ 
conductas delictivas se encuentran factores internos como externos; en cuanto al primero, se encuentran estados psicológicos y biológicos; en los segundos, están los aspectos familiares, económicos, relación con pares, exposición y disposición de las drogas (Esbec y Echeburúa, 2016; Saravia et al., 2014).

Dentro de los factores individuales para el consumo de drogas se ha encontrado una relación directa y positiva entre la impulsividad y el Sistema de Inhibición Conductual (BIS, por sus siglas en inglés), así como el Sistema de Activación Conductual (BAS, por sus siglas en inglés) (Pulido et al., 2016). Pulido, et al. (2016) encontraron que un sistema BIS disminuido y un sistema BAS aumentado desarrollan adicciones, además, el sistema BAS aumentado potencializa la impulsividad. Conjuntamente, existen indicadores que sugieren una relación general entre la motivación hacia el incentivo positivo y la personalidad; es decir, el deseo de consumir sustancias adictivas sería un estado motivacional de incentivo que puede ser explicado por la sobreactivación del BAS, debido a que esta permitiría que la persona se esfuerce más en realizar acciones cuyo fin es la recompensa, con independencia de las posibles consecuencias negativas que implica la conducta (Martínez et al., 2012).

De esta forma, se identifica al sistema BIS como una susceptibilidad al castigo y se relaciona con el estado de ansiedad, y, por el contrario, el sistema BAS se asocia con la susceptibilidad a la recompensa y la impulsividad (Barranco et al., 2009). Las diferencias individuales están asociadas a una alta reactividad del BAS, con la orientación a la recompensa y la impulsividad, o una alta reactividad del BIS, con una propensión a la preocupación y la rumiación ansiosa (Becerra, 2010). Los individuos altamente impulsivos tienen un sistema BAS muy reactivo frente a los menos impulsivos (Pérez et al., 2015). Asimismo, se ha encontrado que la búsqueda de sensaciones tiene relación mediadora con el consumo de alcohol (González-Iglesias et al., 2014).

Por otra parte, otros autores han estudiado de manera separada (del BIS/BAS) a la impulsividad; esta se puede entender como un estilo cognitivo que se caracteriza por tener un pobre autocontrol de la conducta, tomar decisiones de manera apresurada y sin tomar en cuenta las consecuencias (Celma, 2015; RiañoHernández et al., 2015). La impulsividad se asocia a un incremento de la conducta antisocial y el consumo de drogas (Martínez-Fernández et al., 2018).

En población penitenciaria se han encontrado índices altos de impulsividad $y$ búsqueda de sensaciones en comparativa con población general (Betancourt y García, 2015; Santana-Campas, et al., 2019). También, se ha encontrado que los puntajes altos en impulsividad tienen relación con alteraciones en la regulación de la emoción, conducta y cognición relacionadas con conductas antisociales, así como en la 
vulnerabilidad y el mantenimiento en el abuso de drogas; de esta manera, un puntaje alto en impulsividad dificulta la toma de decisiones, toda vez que no permite que estas se tomen en coherencia con el área cognitiva y emocional (Alcázar-Córcoles et al., 2015, Alcázar et al., 2015).

Los varones son más impulsivos $\mathrm{y}$ buscadores de emociones, aventuras y experiencias que las mujeres. Se encontró que el $22.5 \%$ de los adolescentes que consumen Cannabis puntúan más alto en búsqueda de emociones y aventuras, en búsqueda de experiencias y en desinhibición que los no consumidores. Además, aparece un incremento de la impulsividad disfuncional y de la susceptibilidad al aburrimiento asociada al consumo en las mujeres (Pérez de AlbénizGarrote et al., 2019).

Dentro de los sistemas BIS y BAS se ha estudiado la impulsividad como una dimensión dentro de dichos sistemas y se ha encontrado que son potencializadores de la impulsividad, pero los resultados no han sido consistentes. Al respecto, Halty et al. (2017) han mencionado que a pesar de ser una teoría parsimoniosa tiene algunas limitaciones, puesto que no permite distinguir rasgos de manera diferenciada (impulsividad, agresividad, estabilidad o inestabilidad emocional, entre otros) o tipos de personalidad, por lo que resulta necesario estudiar la impulsividad y los sistemas BIS y BAS por separado para delimitar y establecer la relación directa entre estos y el consumo de drogas en personas privadas de la libertad (PPL).

Es por esto que en esta investigación se decidió estudiar como variables independientes la impulsividad y los sistemas BIS/BAS para describir el efecto de estas sobre el consumo de drogas. En cuanto a la búsqueda de sensaciones, Alcázar et al. (2015) comentaron que estas también están relacionadas con las conductas prosociales o las conductas neutras, por lo que se requiere mayor número de investigaciones para describir la relación entre la búsqueda de sensaciones y el consumo de drogas en población penitenciaria.

Puesto que se ha encontrado que la impulsividad es un rasgo que se asocia no solo al consumo de drogas, sino que es un rasgo distintivo de los sujetos que no respetan normas, tienen problemas para el control de impulsos, suelen ser reactivos ante estímulos hostiles y comportamiento criminal, resulta necesario estudiar la impulsividad como variable independiente. Aunado a lo anterior, los problemas de conductas antisociales e impulsividad suelen potencializar el consumo de drogas. Por este motivo y ante la falta de evidencia de lo expuesto en población penitenciaria fue que en la presente investigación se planteó el siguiente objetivo de investigación: determinar la relación de los sistemas BIS/BAS e impulsividad en el consumo de drogas en personas privadas de la libertad. 


\section{Método}

Participantes

Investigación cuantitativa, no experimental, transversal y correlacional. Participaron 175 personas privadas de la libertad en una cárcel de mediana seguridad del Estado de Jalisco, México. Los criterios de inclusión fueron que los participantes supieran leer, escribir y que aceptaran participar en el estudio. El muestro fue no probabilístico, debido a que por medidas de seguridad de los centros penitenciarios no fue posible que proporcionarán datos de las PPL para realizar un muestreo aleatorio.

\section{Instrumentos}

Para medir los Sistemas de Inhibición Conductual y Activación Conductual se utilizaron las Escalas BIS/BAS (Carver y White, 1994), validadas para México por Jiménez et al. (2009). Está compuesta por 24 ítems (4 de relleno: 1, 6, 11, 17). La BIS se compone de 7 ítems $(2,8,13,16,19,22$, $24)$, esta identifica los motivos de evitación o alejamiento de algo desagradable. La escala BAS está formada por tres dimensiones: 1) pulsión, con 4 ítems $(3,9,12,21)$, esta identifica la fuerza que lleva al individuo a conseguir sus objetivos; 2) búsqueda de diversión, placer, o sensaciones (BDP), con 4 ítems $(5,10,15,20)$, que miden las acciones encaminadas a la satisfacción personal, y 3) responsividad a la recompensa (RR), con 5 ítems $(4,7,14,18,23)$, que miden las conductas específicas que llevan a obtener una recompensa.

La forma de contestar la escala BIS/BAS es en escala Likert, que va desde "para mí es 118 PSICỤMEX muy cierto" hasta "para mí es muy falso" y se es muy cierto" hasta 4 que corresponde a "para mí es muy falso". Dos ítems se invierten (2 y 22). Los coeficientes alfa para RR, $P, y$ BDP, son $.73, .76$ y .66 , respectivamente, y para la EBIS es .74. (Carver y White, 1994). Para obtener los resultados se suman los puntos por cada escala, y para un resultado general su suman todas las puntuaciones (Jiménez et al., 2009). Para determinar los puntajes altos, medios y bajos se calcularon mediante las medidas de tendencia central y dispersión.

Para valorar la impulsividad se utilizó la Escala de impulsividad (EI) (Plutchik y Van Praag, 1989), validada para los países de El Salvador, México y España (Alcázar-Córcoles et al., 2015). Esta escala consta de 15 reactivos con cuatro opciones de respuesta: nunca, a veces, a menudo y casi siempre. El resultado posible es de 0 a 45 puntos, el punto de corte es de 20 puntos (Rubio et al., 1998), para determinar "baja impulsividad" se agruparon los resultados de 0 a 19 puntos, para "alta impulsividad" los resultados de 20 a 45 puntos. Además, permite identificar cuatro dimensiones: 1) autoconcepto impulsivo (ítems: 2, 3, 5, 8 y 10), 2) emociones e impulsos primarios (ítems: 7, 9, 12 y 14), 3) planificación (ítems: 4, 11 y 15) y 4) concentración (ítems: 1, 6 y 13). Para identificar variables sociodemográficas y consumo de drogas se construyó un instrumento ex profeso, en el que se incluyeron reactivos para identificar, estado civil, apoyo familiar recibido, tiempo en reclusión, situación jurídica, antecedentes psiquiátricos, antecedentes de sufrir violencia, consumo de drogas del entrevistado y de sus padres. 


\section{Procedimiento}

Esta investigación se enmarca en una investigación general que tiene como objetivo identificar las variables asociadas y predictivas del consumo y tratamiento de drogas, y del riesgo de suicidio en PPL. Para la investigación que nos ocupa, una vez que se contó con las autorizaciones necesarias por parte de las autoridades penitenciarias se procedió a la aplicación de los instrumentos de manera grupal a papel y lápiz en las aulas del área escolar del reclusorio. Los grupos fueron de aproximadamente 20 personas. En total fueron 48 reactivos y la duración aproximada para contestarlos fue de diez minutos.

En cuanto a ética, como ya se mencionó, se contó con el aval de las autoridades penitenciarias y del Consejo Estatal Contra las Adicciones de Jalisco mediante oficio: JAL031/P-01/01042019. Asimismo, se vigiló el cumplimiento del Código de Ética y Conducta de los Servidores Públicos de la Administración Pública del Estado de Jalisco y protección de datos personales. Además, la participación de las PPL fue libre y voluntaria, antes de contestar los cuestionarios, los que decidieron participar firmaron el consentimiento informado.

\section{Tipo de análisis}

Se realizaron análisis descriptivos $\mathrm{y}$ bivariantes, para el tamaño del efecto se calculó la $d$ de Cohen se utilizó la interpretación de Funder y Ozer (2019) donde $d=.10$ efecto pequeño, $d=.20$ medio, $d=.30$ grande y $d=.40$ muy grande; para valorar la asociación entre las variables, se utilizó regresión logística simple.
La regresión logística binomial se realizó con el método INTRODUCIR. Se calcularon los odds ratio $(\mathrm{OR})$ con un intervalo de confianza del 95 \% (IC95 \%). Para el ajuste del modelo se tomó la prueba de Hosmer-Lemeshow y los coeficientes de determinación R2 de Cox y Snell y R2 de Nagelkerke.

La evaluación de fiabilidad de las pruebas se calculó mediante el Alfa de Cronbach. En los análisis se consideró un valor de significancia de $5 \%$. Los análisis se realizaron con el paquete Statistical Package for the Social Sciences versión 24 (SPSS Inc., Chicago, IL, USA).

\section{Resultados}

La muestra final fue de 175 PPL, la media de edad fue de $36, D E=9$; el estado civil fue: $35.4 \%$ solteros, $27.7 \%$ concubinato y 18.3 $\%$ casados. Escolaridad: $50.3 \%$ educación primaria, $24.6 \%$ educación secundaria y $22.3 \%$ educación media superior. E1 85.1\% ya contaban con una sentencia definida (sentenciados) y el $14.9 \%$ estaban en espera de recibir sentencia (procesados). Los delitos con mayor porcentaje fueron el robo (40.6\%) y el homicidio $(31.4 \%)$. La media de tiempo que llevaban en reclusión fue de seis años $(D E=4)$. En cuanto al consumo de drogas, el $68 \%$ de los PPL evaluados reportó consumo antes de ingresar a reclusión.

En la Tabla 1, se describen los resultados de las variables de antecedentes de consumo de drogas por parte del sujeto de estudio y de los padres, así como también, los antecedentes de violencia, tratamiento psiquiátrico y apoyo recibido durante el encarcelamiento. 
Tabla 1

Descriptivo de variables antecedentes y consumo de drogas

\begin{tabular}{ccc}
\hline & $\mathrm{n}$ & $\%$ \\
\hline $\begin{array}{ccc}\text { Violencia en la infancia } \\
\text { No }\end{array}$ & 120 & 68.60 \\
Sí & 55 & 31.40 \\
Consumo de drogas & 56 & 32.00 \\
No & 119 & 68.00 \\
Sí & & \\
Consumo de drogas de padres & 158 & 90.30 \\
No & 17 & 9.70 \\
Sí & & \\
Medicamento psiquiátrico & 161 & 92.00 \\
No & 14 & 8.00 \\
Sí & & \\
Apoyo familiar & 149 & 85.10 \\
Sí & 26 & 14.90 \\
No &
\end{tabular}

Nota: $\mathrm{n}=$ es igual a número de caso. $\%$ = resultado perceptual de la variable descrita

De la Tabla 1 , se resalta que el $68 \%$ de los PPL evaluados reportaron consumo de drogas, que el $85.1 \%$ reportó que recibe algún tipo de apoyo de su grupo familiar y que el $31.4 \%$ reportó que durante su infancia y adolescencia vivió algún tipo de violencia. En cuanto a los resultados descriptivos de los BIS/BAS y la impulsividad, el primero (sistema de inhibición conductual), el $17.1 \%$ puntuó alto; en cuanto al BAS (sistema de activación conductual) el 17.7 $\%$ de los encuestados puntuaron alto, el $32.6 \%$ reportó puntajes altos en la impulsividad (Escala de impulsividad de Plutchik). Para mayor profundidad de los resultados, en la Tabla 2, se describen los resultados de las dimensiones de los sistemas BIS (inhibición conductual, activación conductual e impulsividad) y BAS (pulsión, búsqueda de sensaciones y responsividad recompensa) y de la escala de impulsividad (autoconcepto impulsivo, emociones e impulsos primarios, planificación y concentración). 


\section{Tabla 2}

Resultados descriptivos de las variables independientes

\begin{tabular}{|c|c|c|}
\hline & $\mathrm{N}$ & $\%$ \\
\hline \multicolumn{3}{|c|}{ Inhibición conductual } \\
\hline Baja & 145 & 82.90 \\
\hline Alta & 30 & 17.10 \\
\hline \multicolumn{3}{|c|}{ Activación conductual } \\
\hline Baja & 144 & 82.30 \\
\hline Alta & 31 & 17.70 \\
\hline \multicolumn{3}{|c|}{ Impulsividad } \\
\hline Baja & 118 & 67.40 \\
\hline Alta & 57 & 32.60 \\
\hline \multicolumn{3}{|l|}{ Pulsión } \\
\hline Baja & 145 & 82.90 \\
\hline Alta & 30 & 17.10 \\
\hline \multicolumn{3}{|c|}{ Búsqueda de sensaciones } \\
\hline Baja & 145 & 82.90 \\
\hline Alta & 30 & 17.10 \\
\hline \multicolumn{3}{|c|}{ Responsividad recompensa } \\
\hline Baja & 142 & 81.10 \\
\hline Alta & 33 & 18.90 \\
\hline \multicolumn{3}{|c|}{ Autoconcepto impulsivo } \\
\hline Baja & 146 & 83.40 \\
\hline Alta & 29 & 16.60 \\
\hline \multicolumn{3}{|c|}{ Emociones e impulsos primarios } \\
\hline Baja & 151 & 86.30 \\
\hline Alta & 24 & 13.70 \\
\hline \multicolumn{3}{|c|}{ Planificación } \\
\hline Baja & 144 & 82.30 \\
\hline Alta & 31 & 17.70 \\
\hline \multicolumn{3}{|c|}{ Concentración } \\
\hline Baja & 155 & 88.60 \\
\hline Alta & 20 & 11.40 \\
\hline
\end{tabular}

Nota: $\mathrm{n}=$ es igual a número de caso. \% = resultado perceptual de la variable descrita 
En la Tabla 3, se describen las variables consideradas como antecedentes y tomando como variable dependiente el consumo de drogas, en esta se resalta que para el consumo de drogas el haber sufrido violencia en la infancia fue significativa $(p=.001)$ y el tamaño del efecto fue medio $(\mathrm{d}=.214)$. No tuvieron relación significativa ni efecto el resto de las variables (consumo de drogas por parte de los padres, estar tomando medicamento psiquiátrico y recibir apoyo familiar).

\section{Tabla 3}

Descriptivos de variables de antecedentes por consumo de drogas

\begin{tabular}{|c|c|c|c|c|c|c|}
\hline & \multicolumn{2}{|c|}{ No } & \multicolumn{2}{|c|}{ Sí } & \multirow[t]{2}{*}{$p$} & \multirow[t]{2}{*}{$d$} \\
\hline & $\mathrm{n}$. & $\%$ & $\mathrm{n}$ & $\%$ & & \\
\hline \multicolumn{7}{|c|}{ Violencia en la infancia } \\
\hline No & 49 & 87.50 & 71 & 59.70 & \multirow{2}{*}{$.001^{* * *}$} & \multirow{2}{*}{$.214++$} \\
\hline Sí & 7 & 12.50 & 48 & 40.30 & & \\
\hline \multicolumn{7}{|c|}{ Consumo drogas de padres } \\
\hline No & 53 & 94.60 & 105 & 88.20 & \multirow{2}{*}{.182} & \multirow{2}{*}{.043} \\
\hline Sí & 3 & 5.40 & 14 & 11.80 & & \\
\hline \multicolumn{7}{|c|}{ Medicamento psiquiátrico } \\
\hline No & 52 & 92.90 & 109 & 91.60 & \multirow{2}{*}{.774} & \multirow{2}{*}{.008} \\
\hline Sí & 4 & 7.10 & 10 & 8.40 & & \\
\hline \multicolumn{7}{|c|}{ Apoyo familiar } \\
\hline Sí & 50 & 89.30 & 99 & 83.20 & \multirow{2}{*}{.290} & \multirow{2}{*}{.042} \\
\hline No & 6 & 10.70 & 20 & 16.80 & & \\
\hline
\end{tabular}

En la Tabla 2, se describieron los resultados de las dimensiones de los sistemas BIS y BAS, y de la escala de impulsividad. Ahora en la Tabla 4 , se presentan los resultados de las dimensiones de las variables antes mencionadas por el consumo de drogas. De estos resultados resalta que solo el autoconcepto impulsivo de la escala de impulsividad fue significativo y el efecto fue discreto. 


\section{Tabla 4}

Descriptivos de variables independientes (por dimensiones) por consumo de drogas

\begin{tabular}{|c|c|c|c|c|c|c|}
\hline & \multicolumn{2}{|c|}{ No } & \multicolumn{2}{|c|}{ Sí } & \multirow{2}{*}{$\mathrm{p}$} & \multirow{2}{*}{$\mathrm{D}$} \\
\hline & $\mathrm{n}$ & $\%$ & $\mathrm{n}$ & $\%$ & & \\
\hline \multicolumn{7}{|c|}{ Autoconcepto impulsivo } \\
\hline Baja & 53 & 94.60 & 93 & 78.20 & \multirow{2}{*}{$.006^{* *}$} & \multirow{2}{*}{$0.116+$} \\
\hline Alta & 3 & 5.40 & 26 & 21.80 & & \\
\hline \multicolumn{7}{|c|}{ Emociones e impulsos primarios } \\
\hline Baja & 51 & 91.10 & 100 & 84.00 & \multirow{2}{*}{.207} & \multirow{2}{*}{0.049} \\
\hline Alta & 5 & 8.90 & 19 & 16.00 & & \\
\hline \multicolumn{7}{|c|}{ Planificación } \\
\hline Baja & 45 & 80.40 & 99 & 83.20 & \multirow{2}{*}{.647} & \multirow{2}{*}{0.020} \\
\hline Alta & 11 & 19.60 & 20 & 16.80 & & \\
\hline \multicolumn{7}{|c|}{ Concentración } \\
\hline Baja & 52 & 92.90 & 103 & 86.60 & \multirow{2}{*}{.222} & \multirow{2}{*}{0.043} \\
\hline Alta & 4 & 7.10 & 16 & 13.40 & & \\
\hline \multicolumn{7}{|l|}{ Pulsión } \\
\hline Baja & 45 & 80.40 & 100 & 84.00 & \multirow{2}{*}{.547} & \multirow{2}{*}{0.026} \\
\hline Alta & 11 & 19.60 & 19 & 16.00 & & \\
\hline \multicolumn{7}{|c|}{ Búsqueda de sensaciones } \\
\hline Baja & 43 & 76.80 & 102 & 85.70 & \multirow{2}{*}{.144} & \multirow{2}{*}{0.063} \\
\hline Alta & 13 & 23.20 & 17 & 14.30 & & \\
\hline \multicolumn{7}{|c|}{ Responsividad recompensa } \\
\hline Baja & 45 & 80.40 & 97 & 81.50 & \multirow{2}{*}{.855} & \multirow{2}{*}{0.008} \\
\hline Alta & 11 & 19.60 & 22 & 18.50 & & \\
\hline
\end{tabular}

Nota: Para el cálculo del tamaño del efecto se utilizó la prueba $d$ de Cohen. El Valor de $p$ se calculó mediante la prueba $X^{2}$

+ discreto ++ medio +++ grande ++++ muy grande

$p<.05^{*} p<.01^{* *} p<.001^{* * *}$

Antes de realizar la regresión logística se analizó la relación binaria y estimación de riesgo entre la impulsividad, sistemas BIS/ BAS y el consumo de droga. La única relación positiva y significativa fue la de impulsividad con consumo de drogas $(p=.001 \mathrm{OR}=3.531 \mathrm{LI}$ $=1.584-\mathrm{LS}=7.871$ ).

Por último, se realizaron 11 modelos de regresión logística. El primero consistió en introducir los resultados totales (dicotómicos) de 
las escalas de impulsividad, sistemas BIS/BAS, estado civil, apoyo familiar recibido, tiempo en reclusión, situación jurídica, medicación psiquiátrica, antecedentes de sufrir violencia, consumo de drogas de los padres, por consumo de drogas; el modelo no fue significativo ( $-2 \mathrm{LL}$ 207.689, Hosmer-Lemeshow: X2 .448 y $p=$ .930. R2 de Cox y Snell .065 y R2 de Nagelkerke
.091), por lo que se tomó la decisión de introducir las dimensiones de cada una de las escalas para evaluar el modelo. El modelo de mejor ajuste se presenta en la Tabla 5; en esta, se observa que existen variables que no son significativas, pero al sacarlas del modelo baja el nivel explicativo (r2) por lo que se tomó la decisión de dejarlas dentro del este.

\section{Tabla 5}

Modelo de regresión logística, dimensiones de los sistemas BIS/BAS, de la impulsividad, violencia en la infancia, apoyo familiar respecto al consumo de drogas en mexicanos privados de la libertad

\begin{tabular}{lrrrrrrrr}
\hline \multicolumn{1}{c}{ Variables exposición } & B & $\begin{array}{c}\text { Error } \\
\text { estándar }\end{array}$ & Wald & gl & Sig. & OR & \multicolumn{2}{c}{ C.I. 95\% } \\
& & & & & & & \\
Inferior & Superior \\
\hline Autoconcepto impulsivo & 1.628 & 0.715 & 5.191 & 1 & $.023^{*}$ & 5.094 & 1.255 & 20.667 \\
Violencia en la infancia & 1.589 & 0.47 & 11.431 & 1 & $.001^{* * *}$ & 4.901 & 1.95 & 12.314 \\
Recibe visitas & 0.596 & 0.546 & 1.194 & 1 & .275 & 1.815 & 0.623 & 5.291 \\
Consumo drogas padres & 0.492 & 0.72 & 0.467 & 1 & .494 & 1.636 & 0.399 & 6.716 \\
Pulsión & 0.481 & 0.507 & 0.9 & 1 & .343 & 1.617 & 0.599 & 4.366 \\
Concentración & 0.128 & 0.698 & 0.034 & 1 & .854 & 1.137 & 0.29 & 4.464 \\
Planificación & -0.049 & 0.458 & 0.011 & 1 & .915 & 0.952 & 0.388 & 2.338 \\
Responsividad recompensa & -0.216 & 0.469 & 0.211 & 1 & .646 & 0.806 & 0.321 & 2.022 \\
Emociones e impulsos & -0.334 & 0.661 & 0.255 & 1 & .613 & 0.716 & 0.196 & 2.617 \\
Búsqueda de sensaciones & -0.64 & 0.481 & 1.769 & 1 & .184 & 0.527 & 0.205 & 1.354 \\
Constante & 0.157 & 0.267 & 0.348 & 1 & .555 & 1.171 & & \\
\hline
\end{tabular}

Nota: La variable dependiente consumo de drogas, se categorizó 1 con consumo y 0 sin consumo. OR, odds ratio; IC95 \%, intervalo de confianza al 95 \%. -2LL 187.216, Hosmer-Lemeshow: X² 26.155 y $p=$ .001. R2 de Cox y Snell .168. R2 de Nagelkerke .235.

${ }^{*} p<, 05^{* *} p<, 01{ }^{* * *} p<, 001$

El modelo de regresión logística explica el $23.5 \%$ de la varianza del consumo de drogas en personas privadas de la libertad, destacando que las PPL que sufrieron violencia en la infancia tienen hasta 4.90 veces más de consumo de drogas y autoconcepto impulsivo 5.09 veces más de consumo de drogas. Las variables de estado civil, tiempo en reclusión, situación jurídica y 
medicación psiquiátrica no fueron significativas en este modelo por lo que, en esta muestra, se destaca el efecto sobre el consumo de drogas en personas privadas de la libertad.

\section{Discusión}

Esta investigación tuvo como objetivo principal determinar la relación de los sistemas BIS/BAS e impulsividad en el consumo de drogas en personas privadas de la libertad. El resultado sobresaliente fue que el Sistema de Inhibición Conductual (BIS) y el Sistema de Activación Conductual (BAS) en sus puntuaciones totales no tuvieron una relación directa y significativa con el consumo de drogas, pero sí con la impulsividad, consumo de drogas por parte de los padres y el haber sufrido violencia en la infancia y adolescencia.

En la muestra estudiada se encontró que el $68 \%$ reportó consumo de drogas antes de ingresar a la cárcel y el 9.7 \% refirió consumo de drogas por parte de los padres, de esto último se desprende que a mayor exposición y disposición mayor será el riesgo de consumo de drogas. Esto es consistente con lo reportado por Esbec y Echeburúa (2016) y Saravia et al. (2014). Además, el consumo de drogas también es un predictor de conductas delictivas (Santana-Campas, Hidalgo-Rasmussen y Santoyo, 2019) y trastornos psiquiátricos (Larrotta et al., 2017) de aquí que en la muestra estudiada el $8 \%$ reportó estar en tratamiento psiquiátrico.

En los resultados descriptivos de los sistemas BIS/BAS se encontró que el $17.1 \%$ puntuó alto en BIS y el $17.7 \%$ en BAS, en lo que respecta a la impulsividad el $32.6 \%$ puntuó alto, pero en la regresión logística no fueron significativos los sistemas BIS/BAS en el modelo. En cuanto a esto Celma (2015), RiañoHernández et al. (2015) han recomendado el estudio por separado de estos sistemas y de la impulsividad, de aquí la decisión de analizarlos por sus dimensiones y poder discriminar el efecto de estos sistemas en el consumo de drogas en personas privadas de la libertad.

Al realizar análisis descriptivos por dimensiones, la impulsividad y los sistemas BIS/BAS se encontró que las puntuaciones más altas fueron para las dimensiones de BAS y de la impulsividad (Tabla 2). Posterior a esto, se analizó la relación de estas dimensiones por consumo de drogas, de estos se desprende que solo el autoconcepto impulsivo (dimensión de la impulsividad) tuvo una relación significativa ( $p=.006)$ y efecto discreto $(d=.116)$ sobre el consumo de drogas (Tabla 4). Esto es consistente con lo reportado por Pérez et al. (2015), SantanaCampas, Hidalgo-Rasmussen y AlcázarCórcoles (2019), es decir, la impulsividad y la búsqueda de sensaciones tienen efecto sobre el consumo de drogas y comportamiento delictivo, asumiendo que el BAS orienta a la recompensa (consumo de la droga) y la impulsividad a la búsqueda del estímulo (la droga) (Becerra, 2010).

En cuanto a la función potencializadora de los sistemas BIS/BAS sobre la impulsividad (Pérez et al., 2019), en esta investigación se 
discrimina dicho efecto, toda vez que el sistema BIS no tuvo efecto sobre el consumo de drogas y la impulsividad, solo fueron significativas las dimensiones del BAS y de la impulsividad (Halty et al., 2017). Por tanto, de los resultados de esta investigación se concluye que la pulsión, concentración, planificación, responsividad recompensa, emociones e impulsos, búsqueda de sensaciones, autoconcepto impulsivo, violencia en la infancia, recibe visitas, consumo de drogas por parte de los padres explican en un $23.5 \%$ la variabilidad del consumo de drogas en personas privadas de la libertad.

De esto se resalta que tienen mayor riesgo de consumo de drogas aquellos con autoconcepto impulsivo $(\mathrm{OR}=5.09)$, violencia en la infancia $(\mathrm{OR}=4.90)$, recibe visitas $(\mathrm{OR}=1.81) \mathrm{y}$ consumo de drogas por parte de los padres (OR $=1.63)$, respecto a los que no presentan estos indicadores. Es decir, las personas privadas de la libertad con autoconcepto impulsivo tienen 5.09 veces más de riesgo para el consumo de drogas y 4.9 veces más de los que reportan violencia en la infancia.

De lo anterior se desprende que la disposición, exposición a la droga y haber sido víctima de violencia en la infancia y adolescencia son factores de riesgo para el consumo de drogas; con esto se discrimina el efecto del sistema BIS y se resalta el efecto del sistema BAS y el autoconcepto impulsivo sobre el consumo de drogas en personas privadas de la libertad. Los resultados de investigación pueden tener implicaciones terapéuticas para el abordaje de las adicciones en el contexto penitenciario, es decir, que se tendrían que estar tomando en cuenta, para el tratamiento, intervenciones enfocadas a la disminución de la impulsividad, al control del sistema BAS (pulsión, búsqueda de sensaciones, responsividad recompensada) y a sanar heridas de la infancia.

\section{Conflictos de interés}

Los autores declaran no tener ningún conflicto en relación con el tema tratado en la presente investigación.

\section{Referencias}

Alcázar, M.A., Verdejo, A., Bouso, J.C. y Ortega, J. (2015). Búsqueda de sensaciones y conducta antisocial. Anuario de Psicología Jurídica 25, 75-80. http:// dx.doi.org/10.1016/j.apj.2015.01.003

Alcázar-Córcoles, M., Verdejo, A. y BousoSáiz, J. (2015). Propiedades psicométricas de la escala de impulsividad de Plutchik en una muestra de jóvenes hispanohablantes. Actas Españolas de Psiquiatría, 43(5), 161-9. https://www.actaspsiquiatria.es/repositorio/17/97/ESP/17-97ESP-161-9-505471.pdf

Barranco, L., Rodarte, B., Medina, Y. y Solís-Cámara, P. (2009). Evaluación psicométrica de los sistemas de activación e inhibición del comportamiento en adultos mexicanos. Anales de Psicología, 25(2), 358-367. http://www.redalyc.org/ $\mathrm{html} / 167 / 16712958019 /$ 
Beatriz González-Iglesias, B., Gómez-Fraguela, J.A., Gras, M.E. y Planes, M. (2014). Búsqueda de sensaciones y consumo de alcohol: El papel mediador de la percepción de riesgos y beneficios. Anales de psicología, 30(3), 1061-1068. http:// dx.doi.org/10.6018/analesps.30.3.170831

Becerra, J. (2010). Actividad de los sistemas de aproximación e inhibición conductual y psicopatología. Anuario de Psicología Clínica y de la Salud, 6, 61-65. http:// institucionales.us.es/apcs/doc/APCS_6_ esp_61-65.pdf

Betancourt, D. y García, S. (2015). La impulsividad y la búsqueda de sensaciones como predictores de la conducta antisocial en adolescentes. Enseñanza e Investigación en Psicología, 20(3), 309-315. https:/www.redalyc.org/ pdf/292/29242800008.pdf

Castellanos, G. (2015). Detección de drogas y tratamiento de adicciones en dos centros penitenciarios de la Ciudad de México. Integración Académica en Psicología, 3(7). http://integracion-academica.org/attachments/article/88/08\%20Drogas\%20 Adicciones\%20-\%20GE\%20Castellanos. pdf

Celma, J. (2015). Bases teóricas y clínicas del comportamiento impulsivo. Ediciones San Juan de Dios - Campus Docent. https://bibliosjd.files.wordpress. com/2015/02/bases_teoricas_y_clinica_ comportamiento_impulsivo.pdf
Comisión Nacional contra las Adicciones (2017). Encuesta nacional de consumo de drogas, alcohol y tabaco, ENCODAT 2016-2017.https://drive.google.com/ file/d/1zIPBiYB3625GBGIW5BX0TT_ YQN73eWhR/view

Esbec, E. y Echeburúa, E. (2016). Abuso de drogas y delincuencia: consideraciones para una valoración forense integral. Adicciones, 28(1), 48-56. http://www. adicciones.es/index.php/adicciones/ article/view/790

Funder, D. y Ozer, D. (2019). Evaluating Effect Size in Psychological Research: Sense and Nonsense. Advances in Methods and Practices in Psychological Science, 2(2), 156 -168. https://doi. org/10.1177/2515245919847202

Halty, L., González, J.L. y Andrés Sotoca, A. (2017). Modelo ENCUIST: aplicación al perfilado criminal. Anuario de psicología jurídica, 27(1), 21-31. https://doi. org/10.1016/j.apj.2017.03.001

Larrotta, R., Rangel, K., Luzardo, M., Gómez, N.y Redondo,J.(2017). Consumo de sustancias psicoactivas antes y después del ingreso a prisión en catorce centros carcelarios de Colombia. Revista Colombiana de Ciencias Sociales, 8(1), 24-44. https://doi. org/10.21501/22161201.1900

Martínez, M., Zalazar, J., Pilatti, A. y Cupani, M. (2012). Adaptación del cuestionario de personalidad BIS BAS IPIP a una muestra de estudiantes universitarios argentinos 
$\mathrm{y}$ análisis de su relación con patrones de consumo de alcohol. Avances en Psicología Latinoamericana, 30(2), 304316. https://www.redalyc.org/articulo. oa? id=79924881007

Martínez-Fernández, V., Lloret-Irles, D. y Segura-Heras, J. (2018). Impulsividad y búsqueda de sensaciones como predictores del consumo de drogas en adolescentes: un estudio longitudinal. Revista de Psicología Clínica con Niños $y$ Adolescentes, 5(3), 9-15. https://doi. org/10.21134/rpcna.2018.05.3.1

Organización Mundial de la Salud (2019). El consumo de drogas debe ser tratado como un problema de salud pública, regido por el pleno respeto a los derechos humanos: UNODC. http://www.onu.org. $\mathrm{mx} / \mathrm{el}$-consumo-de-las-drogas-debe-sertratado-como-un-problema-de-saludpublica-regido-por-el-pleno-respeto-alos-derechos-humanos-unodc/

Pérez de Albéniz-Garrote, G., Medina, B. y Rubio, L. (2019). Influencia de la impulsividad y de la búsqueda de sensaciones en el consumo precoz de Cannabis. Diferencias de género y orientaciones para la prevención. Revista Española de Orientación y Psicopedagogía, 30(1), 1.er cuatrimestre, 27-40. http://revistas.uned.es/index.php/ reop/article/view/25192/19995

Pérez, F., Martín-Moreno, C., López, R., Bernabeu, F. y Esteve, Z. (2015).
Sensibilidad al refuerzo y al castigo e impulsividad en criminales muy violentos. Una evaluación psicopatológica cualitativa a partir de varios sujetos condenados. Behavior \& Law Journal, 1(1), 49-62. http://behaviorandlawjournal. com/index.php/BL/article/view/8

Pulido, M., Rivera-Fong, L., Fondón, A. y Vázquez, P. (2016). Propiedades psicométricas de la escala BIS/BAS en una muestra de estudiantes universitarios mexicanos. Enseñanza e Investigación en Psicología, 21(3), 300-310. https://www. redalyc.org/articulo.oa? $\mathrm{id}=29248182010$

Riaño-Hernández, D., Guillén, A. y Buela-Casal, G. (2015). Conceptualización y evaluación de la impulsividad en adolescentes: una revisión sistemática. Universitas Psychologica, 14(3), 1077-1090. http:// dx.doi.org/10.11144/Javeriana.upsy14-3. ceia

Santana-Campas, M.A., Hidalgo-Rasmussen, C.A. y Alcázar-Córcoles, M.A. (2019). La impulsividad y desesperanza, como factores del riesgo de suicidio en jóvenes mexicanos privados de la libertad. Revista Argentina de Clínica Psicológica. 28(4), 544-553. https://doi. org/10.24205/03276716.2019.1132

Santana-Campas, M.A., Hidalgo-Rasmussen, C.A. y Santoyo-Telles, F. (2019). Impulsividad, consumo de drogas, tipo de delito y riesgo de suicidio en jóvenes mexicanos privados de la libertad. Acta Universita- 
ria, 29, e2284. http://doi.org/10.15174. au.2019.2284

Saravia, J., Gutiérrez, C. y Frech, H. (2014). Factores asociados al inicio de consumo de drogas ilícitas en adolescentes de educación secundaria. Revista Peruana de Epidemiología, 18(1), 1-7. https:/www. redalyc.org/pdf/2031/203131355003.pdf

Secretariado Ejecutivo del Sistema Nacional de Seguridad Pública (2019). Incidencia delictiva. https://www.gob.mx/sesnsp/ articulos/incidencia-delictiva

Uceda-Maza, F.X., Navarro-Pérez, J.J. y PérezCosín, J.V. (2016). Adolescentes y drogas: su relación con la delincuencia. Revista de Estudios Sociales, 58, 63-75. http:// journals.openedition.org/revestudsoc/346

Enviado: 04 de mayo de 2020

Revisado: 31 de agosto de 2020

Aceptado: 06 de octubre de 2020 